\title{
Modeling Damping for a Loaded Spring in an Acoustic Liquid Media
}

\section{Cliff Orori Mosiori}

Department of Mathematics and Physics, Technical University of Mombasa, Box 90420 - 80100, Mombasa, KENYA

Corresponding Contact:

Email: corori@tum.ac.ke

\begin{abstract}
Free vibrating motion can take place in an acoustic media. This motion can be steady hence have constant periodic variations or unsteady and thus experience light damping or heavy damping. We give a modeled analysis of unsteady periodic motion of an oscillator in a cylindrical acoustic medium that allow such waves to be transmitted through them. This has been approached by calculating variation within the proposed boundary functions and boundary potentials. Limitations for these calculations have been done depending on the time, and how free oscillations are expected to behave in cylinder carrying a suspended mass. This work investigated motion by constructions that interact with their environment with the acoustic media. Since the dynamics considered here were very complex, modeling the system with one grade of free motion and applying different types of constructions whether ground, underground, cylindrical, spherical constructions and containers was considered. This work borrowed heavily on the modeling of seismic and blast waves as modeled with rigid inclusions containing elastically fastened mass interacting continuous solid medium. This study joined motion of any continuous medium with other discrete systems. The results displayed measurement systems for wave processes having interference at their eigenfrequencies just like those under seismic wave interactions and this work considered the result as similar to those in discrete systems.
\end{abstract}

Key words

Oscillations, Acoustics, free vibrations, discrete system

\section{INTRODUCTION}

Modern engineering and construction industries require the calculation of the effect of structural elements of constructions on the motion of waves in the medium surrounding the body (Huang etal, 1974). The investigations onto the behavior of small systems where the complementary masses are fastened are of great interest in vibratory technology and 
the focus has been on the questions of estimating the damping ability of dynamical system and its impacts (Seyfullayev and Agayeva, 1998). Many studies have been that if the rigidity of the shell is larger than the rigidity of shock absorbers, springs, any shell deformation is always ignored (Seyfullayev etal, 2012). As a consequence, the effect of loads located in the cylindrical shell to the behavior of the shell at the interaction with the spherical wave of pressure need more investigation.

\section{Methodology}

A system of loads having concentrated masses was attached to the inner surface of the shell by help of elastic springs. A steel shell by help of elastic springs was immersed in water. The rigid cylindrical inclusion (a steel shell) was elastically suspended mass in its inner is studied as shown in figure 1 and was assumed to periodically and continuously in the medium which was water.

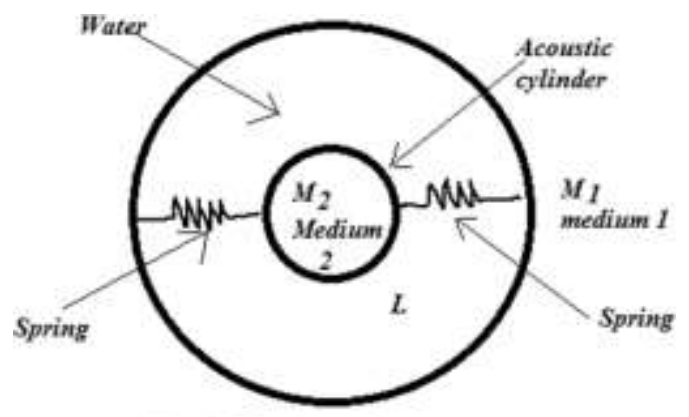

Fig. 1: Experimental set-up

Numerical calculations were then carried out by applying spherical exponentially wave profile. Any diffraction of the generated elastic waves in water having a free surface, though fastened to the platform by a spring and any at the action of the harmonic loading were investigated. The motion the oscillator in this acoustic medium after the passage of the wave was also investigated by using eigenfrequencies (Huang etal, 1974). Investigations were also done to find out if the system can also be in resonance by action of external vibration sources.

\section{Results AND Discussions}

\section{Numerical Analyze}

Due to the complexity of analysis, the system was considered as being flat and he medium as irrotational (Gorshkov and Tarlakovskii, 1990). With this assumption, sound generated has a change in velocity potential in the acoustic cylinder described by the Eq (1):

$$
\Delta \varphi=\frac{1}{a^{2}} \frac{\partial^{2} \varphi}{\partial t^{2}}
$$

where $\Delta$ - Laplace's operator; $a$ is velocity of sound distribution in the medium inside the steel shell, $\varphi$ - is velocity potential in which the operation $\vec{\vartheta}=\operatorname{grad} \varphi$ gives $\vec{\vartheta}$ velocity (Seyfullayev and Agayeva, 1998; Seyfullayev etal, 2012). Assuming also that there no wave transient and hence no diffraction, the waves moves in the same direction with the certain velocity. Applying the principle of relativity for this sound wave, where the medium is not moving but has the velocity of the fluid (Mamedova etal, 2013; Agalarova, 
1997). Then the motion experienced by the medium inside the shell (M2) and that outside the shell $\left(\mathrm{M}_{1}\right)$ can be described by;

$$
\left\{\begin{array}{l}
M_{1} \frac{d^{2} x_{1}}{d t^{2}}=P+L\left(x_{2}-x_{1}\right) \\
M_{2} \frac{d^{2} x_{2}}{d t^{2}}=-L\left(x_{2}-x_{1}\right)
\end{array}\right.
$$

where $M_{1}$ is regarded here as the cage mass while $M_{2}$ is regarded as the mass of the spring body. Equally $x_{1}$ as the replacement of the cage while $x_{2}$ as replacement of the spring body while $L$ as rigidity of the spring and $P$ as the power of the fluid's action on the shell (Seyfullayev etal, 2014; Sinyavskii, 1980). If we take $\rho$-density and $\theta$ - polar angle and since we had a cylinder, then for the cylindrical inclusion with radius $r_{0}$, power of the fluid on the shell can be given as;

$$
P=r_{0} \int_{0}^{2 \pi} p \cos \theta d \theta, \text { where } p=-\rho \frac{\partial \varphi}{\partial t},
$$

Since the springs used were identical, normal components of the velocity to the cage surface are equal (Chen etal, 1976; Seyfullayev and Agayeva, 1998). This means that the components of the velocity of the fluid and the cage can be equated as:

$$
\frac{\partial \varphi}{\partial r}=\frac{d x_{1}}{d t} \cos \theta
$$

Using Eq (1) within the restriction of boundary conditions in Eq (2) and solving Eq (3) and Eq (4) by applying initial conditions as;

$$
\left.\varphi\right|_{t=0}=\left.0 \frac{\partial \varphi}{\partial t}\right|_{t=0}=0
$$

then $\mathrm{Eq}(1)$ in cylindrical coordinates can be expressed as:

$$
\frac{\partial^{2} \varphi}{\partial r^{2}}+\frac{1}{r} \cdot \frac{\partial \varphi}{\partial r}+\frac{1}{r^{2}} \cdot \frac{\partial^{2} \varphi}{\partial \theta^{2}}=\frac{1}{a^{2}} \frac{\partial^{2} \varphi}{\partial t^{2}}
$$

In polar coordinates, a trial solution to $\mathrm{Eq}(6)$ can be given in the form:

$$
\varphi(\mathrm{r}, \theta, \mathrm{t})=\varphi_{1}(\mathrm{r}, \mathrm{t}) \cos \theta
$$

Solving Eq (6) using Laplace's operators get:

$$
\frac{\partial^{2} \varphi_{1}}{\partial r^{2}}+\frac{1}{r} \cdot \frac{\partial \varphi_{1}}{\partial r}-\frac{1}{r^{2}} \cdot \varphi_{1}=\frac{1}{a^{2}} \cdot \frac{\partial^{2} \varphi_{1}}{\partial t^{2}}
$$

If we apply Laplace-Carson transformation to Eq (8) and the condition in eq (5) we get;

$$
\bar{\varphi}_{1}^{\prime \prime}+\frac{1-\bar{\varphi}_{1}^{\prime}}{r}-\left(\frac{p^{2}}{a^{2}}+\frac{1}{r^{2}}\right) \bar{\varphi}_{1}=0
$$

A simplified solution to $\mathrm{Eq}(9)$ requires a restriction condition of infinity in the form of; 
$\overline{\varphi_{1}}=C K_{1}\left(\frac{p r}{a}\right)$

Where $\mathrm{r}$ - distance from the cage center, $\mathrm{K}_{1}$ - Macdonald's functionof the first order. And thus, taking into account Eq (7), it followsfrom Eq (3) that;

$p=-\rho \frac{\partial \varphi_{1}}{\partial t} \cdot \cos \theta$ hence; $P=-\rho r_{0} \frac{\partial \varphi_{1}}{\partial t} \cdot \pi$

Equally, considering the expressions for Eq (11); Eq (4) and Eq (7) into Eq (2), then Eq (2) will take the form:

$$
\left\{\begin{array}{l}
M_{1} \frac{\partial^{2} \varphi_{1}}{\partial r \partial t}+\rho r_{0} \pi \frac{\partial \varphi_{1}}{\partial t}=L\left(x_{2}-x_{1}\right) \\
M_{2} \frac{\partial^{2} x_{2}}{\partial t^{2}}+L\left(x_{2}-x_{1}\right)=0
\end{array}\right.
$$

Using the Laplace- Carson transformation, expressions in Eq (12) can take the form:

$$
\left\{\begin{array}{l}
p M_{1}\left(\bar{\varphi}_{1}^{\prime}-\dot{x}_{0}\right)+\rho r_{0} \pi p \bar{\varphi}_{1}=L\left(\overline{x_{2}}-\overline{x_{1}}\right) \\
M_{2}\left(p^{2} \overline{x_{2}}-p \dot{x}_{0}\right)+L\left(\overline{x_{2}}-\overline{x_{1}}\right)=0
\end{array}\right.
$$

where $\dot{x}_{0}$ is assume to be the initial velocity of the shell. Since we applied the restriction condition of infinity (Seyfullayev and Agayeva, 1998; Agalarova, 1997; Sinyavskii, 1980) to get $\mathrm{Eq}(10)$ then from $\mathrm{Eq}(13)$ we can get $C$ expressed as;

$$
C=\frac{-\left(M_{1} M_{2} p^{2}+M_{1} L+M_{2} L\right) \cdot \dot{x}_{0}}{\left[\left(M_{1} M_{2} p^{2}+M_{1} L+M_{2} L\right) \cdot\left(\frac{p}{a} K_{0}+\frac{1}{r} K_{1}\right)-\rho r_{0} \pi K_{1}\left(M_{2} p^{2}+L\right)\right]}
$$

Where $K_{0}$ is the Macdonald's function of the zero order or we can rewrite Eq (14) in the form of $\mathrm{Eq}(15)$ :

$$
C=\frac{-r_{0} \cdot \dot{x}_{0}}{\frac{p r_{0}}{a} K_{0}+K_{1}+\left(1+\frac{M_{2} L}{M_{1} M_{2} p^{2}+M_{1} L+M_{2} L}\right) \frac{\rho r_{0}^{2} \pi}{M_{1}} K_{1}}
$$

$\mathrm{Eq}$ (15) modifies the original which we can now easily get by equating;

$$
\begin{aligned}
& P K_{0}\left(\frac{p r}{a}\right) \rightarrow \frac{a}{r \sqrt{\left(\frac{a t}{r}\right)^{2}-1}} ; P K_{1}\left(\frac{p r}{a}\right) \dot{\rightarrow} \sqrt{\left(\frac{a t}{r}\right)^{2}-1} ; \\
& \frac{p}{p^{2}+e^{2}} \rightarrow \frac{\sin e t}{e}, \quad t>\frac{r}{a}, \quad e^{2}=\left(\frac{1}{M_{1}}+\frac{1}{M_{2}}\right) L
\end{aligned}
$$


By simplicity, we can let the original denominator by $z$, and $\theta_{1}=\frac{a t}{r_{0}}$ respectively, we obtain;

$$
-z=\frac{1}{\sqrt{\theta_{1}^{2}-1}}+\left(1-\frac{\rho r_{0}{ }^{2} \pi}{M_{1}}\right) \sqrt{\theta_{1}^{2}-1}+\frac{L \rho \pi r_{0}^{2}}{M_{1}^{2} e} \int_{0}^{t} \sin (t-\eta) \cdot e \cdot \sqrt{\left(\frac{a \eta}{r_{0}{ }^{2}}\right)^{2}}-1 d \eta
$$

and by a way of introducing $M_{1}=\rho_{*} r_{0}^{2} \pi, \quad L=M_{2} \omega^{2}, \quad e=\omega \sqrt{\frac{M_{2}}{M_{1}}+1}$ we finally arrive at:

$-z=\frac{1}{\sqrt{\theta_{1}^{2}-1}}+\left(1-\frac{\rho}{\rho_{*}}\right) \sqrt{\theta_{1}^{2}-1}+\frac{\rho}{\rho_{*}} \cdot \frac{\omega r_{0}}{a \sqrt{\frac{M_{1}}{M_{2}}+\left(\frac{M_{1}}{M_{2}}\right)^{2}}} \cdot \int_{1}^{\theta_{1}} \sqrt{\theta_{*}^{2}-1} \sin \frac{r_{0} e}{a}\left(\theta_{1}-\theta_{*}\right) d \theta_{*} \quad\left(15^{a}\right)$

As a modeled expression Eq (15b) can be used to analyze some particular cases for some values of parameters having defined boundary functions.

\section{Numerical Analysis using Defined Boundaries}

\section{CASE 1: Boundary conditions by letting $M_{1}=0$}

We can take the first boundary condition (Seyfullayev etal, 2012) by letting $M_{1}=0$. For this particular case Eq (14) reduced to:

$$
C=\frac{1}{p} \cdot \frac{-\dot{x}_{0}}{\frac{1}{a} K_{0}-p K_{1} \cdot \frac{\rho \pi r_{0}}{L}+\frac{K_{1}}{p}\left(-\frac{\rho \pi r_{0}}{M_{2}}+\frac{1}{r_{0}}\right)}
$$

Following the condition in Eq 16) by denoting the denominator originals as $z$, we get a solution (Huang etal, 1974) expresses as:

$$
z=\frac{1}{a} \cdot \operatorname{Arcch} \frac{a t}{r}-\frac{\rho \pi r_{0}}{L} \cdot \frac{a}{r_{0}} \cdot \frac{t}{\sqrt{t^{2}-\frac{r_{0}^{2}}{a^{2}}}}+\left(-\frac{\rho \pi r_{0}}{M_{2}}+\frac{1}{r_{0}}\right)\left(\frac{a t}{2 r_{0}} \sqrt{t^{2}-\frac{r_{0}^{2}}{a^{2}}}-\frac{r_{0}}{2 a} \operatorname{Arcch} \frac{a t}{r_{0}}\right)
$$

It can also be expressed as;

$$
z=\frac{1}{a} \cdot \operatorname{Arcch} \theta_{1}-\frac{\rho \pi r_{0}}{L} \cdot \frac{\theta_{1}}{\sqrt{\theta_{1}^{2}-1}}+\left(-\frac{\rho \pi r_{0}^{2}}{M_{2}}+1\right)\left(\frac{\theta_{1}}{2 a} \sqrt{\theta_{1}^{2}-1}-\frac{1}{2 a} \operatorname{Arcch} \theta_{1}\right)
$$

where $\theta_{1}=\frac{a t}{r_{0}}$

If we let $\frac{p C(p)}{\dot{x}_{0}} \rightarrow S(t)$, then $\frac{C \cdot p}{\dot{x}_{0}} \cdot \bar{z}=1$, then; we apply the famous Borel's theorem this solutions to obtain:

$\frac{d}{d t} \int_{0}^{t} S(t-\tau) z(\tau) d \tau=1$ 
And by integrating we get :

$\int_{0}^{t} S(t-\tau) z(\tau) d \tau=t+c_{1}$

From the Borel's theorem, for $t \prec \frac{r_{0}}{a}, S=0, \quad z=0$ hence the integral limits are as;

$\int_{\frac{r_{0}}{a}}^{t} S(t-\tau) z(\tau) d \tau=t+c_{1}$

And for $t=\frac{r_{0}}{a}$ the interval is equal to zero resulting into $c_{1}$ is determined as:

$c_{1}=-\frac{r_{0}}{a}$

Mathematically, if we introduce the notation of $t$ as:

$t=\frac{r_{0} \theta_{1}}{a}$

and use it to find the originals of function $\mathrm{C}_{(\mathrm{p})}$ using the integral equation as proposed by Volterra and take as of the first kind by Volterra (Sinyavskii, 1980) then;

$\int_{1}^{\theta_{1}} S\left(\theta_{1}-\tau\right) z(\tau) d \tau=\theta_{1}-1$

CASE 2: Boundary condition by letting $\rho=\rho_{*}, \quad M_{1}=M_{2}, \quad e=\omega \sqrt{2}$ Using Eq (15a) which had the expression given by:

$-z=\frac{1}{\sqrt{\theta_{1}^{2}-1}}+2 \sqrt{\theta_{1}^{2}-1}+\frac{\omega r_{0}}{a \sqrt{2}} \int_{1}^{\theta_{1}} \sqrt{\theta_{*}^{2}-1} \sin \frac{r_{0} \omega \sqrt{2}}{a}\left(\theta_{1}-\theta_{*}\right) d \theta_{*}$

We can let $\frac{\omega r_{0}}{a}=\frac{1}{\sqrt{2}}$ where $\frac{a}{r_{0}}=\omega_{1}$ is an eigen frequency of the medium in $r_{0}$. Having this added conditions, we get $-Z_{n}$ as;

$-z_{n}=\frac{1}{\sqrt{\theta_{1}^{2}-1}}+2 \sqrt{\theta_{1}^{2}-1}+\frac{1}{2} \int_{1}^{\theta_{1}} \sqrt{\theta_{*}^{2}-1} \sin \left(\theta_{1}-\theta_{*}\right) d \theta_{*}$

This expression can further be analyzed numerically by letting $\theta_{1} \approx 1, \quad S \approx \frac{2}{\pi z} S$ is to obtain a potential $\varphi$ expressed as;

$$
\frac{1}{a \dot{x}_{0}} \cdot \varphi_{1}=\int_{r / r_{0}}^{\theta_{1}} \frac{S\left(\theta_{1}-\theta_{*}\right) \theta_{*}}{\sqrt{\theta_{*}^{2}-\frac{r^{2}}{r_{0}^{2}}}} d \theta
$$




\section{SIMULATION METHOD}

Softwares have been developed that analyze coupled oscillation by simulation. In our case, the considered experiment was assumed to be flat and therefore the motion of the fluid is potential. This means that the equation of the motion of the fluid is a wave Eq (1) applies also to simulation approach (Seyfullayev etal, 2014; Agalarova, 1997). In programming the simulator, the area of contact of the fluid with the moving cylinders, the normal components of the velocity of the fluid and the shell are taken as equal. This condition then reduces the experiment as to have harmonic vibrations of the system obeying transcendental equations for its frequencies. These assumptions reduce the problem to a level where the software used the inverse method (Sinyavskii, 1980) i.e. frequency of the system without any fluid (oscillator) is expressed analytically by the frequency of the main system, not solving the transcendental equations. When the software is run, calculation of eigen frequencies and amplitudes of vibrations of the elastic element (Seyfullayev etal, 2012; Seyfullayev and Agayeva, 1998) in the fluid are done. Software was used and the following graphs were obtained. As observed from figure 1 and 2 below, the graphs of dependencies of the boundary function $S$ and potential $\varphi$ on the time for density values taken as $\rho=1$ and 2 and the mass of the vibrating body taken as

$$
m=\frac{1}{M}=2 ; 1 ; \frac{2}{3} ; 0,5 \text {. }
$$

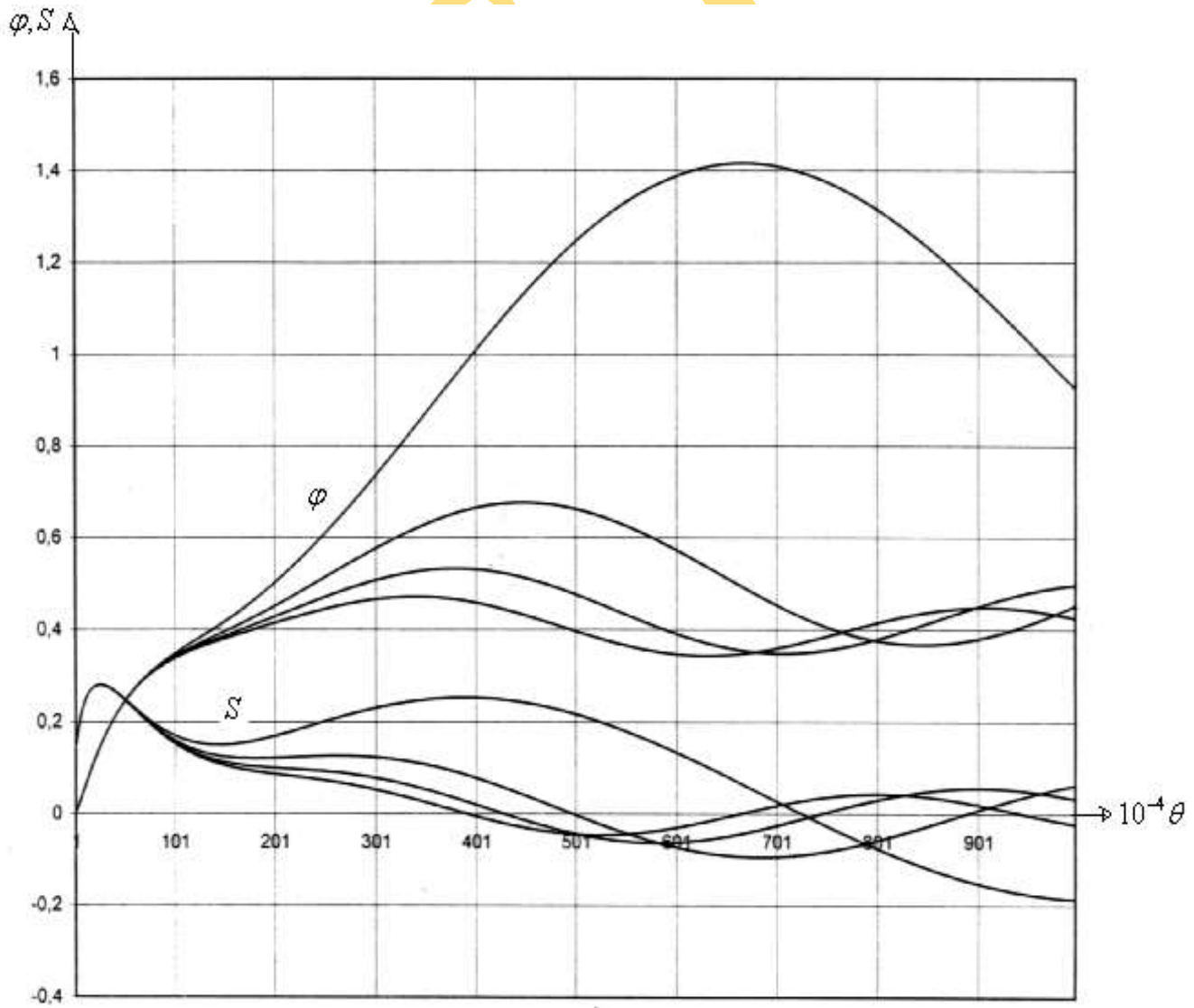

Fig. 3: Boundary Sand potential $\varphi$ with density value $\rho=2$ 
It can be observed from the graphs that function $S$ vibrates after the initial deflection with attenuation approaching to zero, but potential $\varphi$ with vibrations approaches a constant value for each density $\rho$ at different relative internal masses when taken as $\frac{1}{M}$ at small amplitudes (Seyfullayev etal, 2012). Assuming that the medium from the external side is restricted by unmoving surface $\left(r=r_{1}\right)$ or in the case of the unrestricted medium on the surface ( $r=r_{1}$ ) there will be a node of standing wave (Seyfullayev etal, 2014) obtained by a restriction given by;

$\left.\vartheta\right|_{r=r_{1}}=\left.\frac{\partial \varphi}{\partial r}\right|_{r=r_{1}}=0$

In order to find eigen frequencies of the system, we can now apply the method of separation of variables (Mamedova etal, 2013) and this gives a solution of Eq (6) in the form $\mathrm{Eq}(21)$ :

$$
\begin{aligned}
& \varphi(r, \theta, t)=R(r) e^{i \omega t} \cos \theta \\
& x_{1}=B e^{i \omega t} ; \quad x_{2}=C e^{i \omega t}
\end{aligned}
$$

where, B and C are unknown constants to be determined using Eq (21) in Eq (6), such that $\mathrm{Eq}(6)$ takes the form:

$R^{\prime \prime}+\frac{1}{r_{1}} R^{\prime}+\left(1-\frac{1}{r_{1}^{2}}\right) R=0$

where $r=\frac{a}{\omega} r_{1}$

since this expression can also be taken as Eq (12), its solution for Eq (22) can take the form $\mathrm{Eq}(12)$ as:

$R=E J_{1}\left(\frac{\omega r}{a}\right)+D N_{1}\left(\frac{\omega r}{a}\right)$

where $\mathbf{E}$ and $\mathbf{D}$ are also unknown which must be determined. Hence we need to apply the Bessel and Neumann cylindrical functions (Seyfullayev and Agayeva, 1998; Agalarova, 1997). Considering;

$J_{1}\left(\frac{\omega r}{a}\right), N_{1}\left(\frac{\omega r}{a}\right)$

as Bessel and Neumann cylindrical function and solving for expressions Eq (21) by substituting it into Eq (2) and in Eq (4) and by taking into account Eq (3) we easily get the system of algebraic homogeneous equations in relation to constants $\mathrm{E}, \mathrm{B}, \mathrm{C}$, and D stated in $\mathrm{Eq}(21) \mathrm{Eq}(23)$ respectively as:

$\left\{\begin{array}{l}\left(M_{1} \omega^{2}-L\right) B+\rho r_{0} \pi R i \omega+L C=0 \\ L B+C\left(M_{2} \omega^{2}-L\right)=0 \\ R^{\prime}-i \omega B=0 \\ R^{\prime}=0\end{array}\right.$ 
Bessel and Neumann functions require non-trivial solutions (Seyfullayev etal, 2012) of system to Eq (24) and thus we take the main determiner of the named system to zero so as to obtain;

$$
\begin{array}{cccc}
\rho r_{0} \pi i \omega J_{1}\left(\frac{r_{0} \omega}{a}\right) & M_{1} \omega^{2}-L & L & \rho r_{0} \pi i \omega N_{1}\left(\frac{r_{0} \omega}{a}\right) \\
0 & L & M_{2} \omega^{2}-L & 0 \\
J_{1}^{\prime}\left(\frac{r_{0} \omega}{a}\right) & -i \omega & 0 & N_{1}^{\prime}\left(\frac{r_{0} \omega}{a}\right) \\
J_{1}^{\prime}\left(\frac{r_{1} \omega}{a}\right) & 0 & 0 & N_{1}^{\prime}\left(\frac{r_{1} \omega}{a}\right)
\end{array}
$$

This gives a frequency equation of the form of Eq (26) :

$$
\begin{aligned}
& J_{1}^{\prime}\left(\frac{\omega r_{0}}{a}\right) N_{1}^{\prime}\left(\frac{\omega r_{1}}{a}\right)\left(M_{1} M_{2} \omega^{4}-L_{2} \omega^{2}\left(M_{1}+M_{2}\right)\right)-J_{1}^{\prime}\left(\frac{\omega r_{1}}{a}\right) N_{1}^{\prime}\left(\frac{\omega r_{0}}{a}\right)\left(M_{1} M_{2} \omega^{4}-L_{2} \omega^{2}\left(M_{1}+M_{2}\right)\right)- \\
& -J_{1}^{\prime}\left(\frac{\omega r_{1}}{a}\right) N_{1}^{\prime}\left(\frac{\omega r_{0}}{a}\right) \rho \pi r_{0}\left(M_{2} \omega^{2}-L\right) \omega^{2}=0
\end{aligned}
$$

As a rule, we introduce the following denotations to simplify analysis:

$$
\frac{L}{M_{1}}=k_{1}^{2}, \frac{L}{M_{2}}=k_{2}^{2}, \frac{L}{\rho r_{0}^{2} \pi}=k_{0}^{2}, m=\frac{\rho \pi r_{0}}{M_{1}}
$$

With this simplified conditions, Eq (26) takes the following form:

$$
\begin{aligned}
& \left(-J_{1}^{\prime}\left(\frac{\omega r_{1}}{a}\right) N_{1}^{\prime}\left(\frac{\omega r_{0}}{a}\right)\right)\left(\omega^{4}-\omega^{2}\left(-2 k_{3}^{2}\right)+k_{1}^{2} k_{2}^{2}\right)-m \frac{1}{r_{0}}\left(\omega^{4}-\omega^{2} k_{2}^{2}\right)=0 \\
& J_{1}^{\prime}\left(\frac{\omega r_{0}}{a}\right) N_{1}^{\prime}\left(\frac{\omega r_{1}}{a}\right)\left(\omega^{2}-k_{1}^{2}-k_{2}^{2}\right)-J_{1}^{\prime}\left(\frac{\omega r_{1}}{a}\right) N_{1}^{\prime}\left(\frac{\omega r_{0}}{a}\right)\left(\omega^{2}-k_{1}^{2}-k_{2}^{2}\right)-J_{1}^{\prime}\left(\frac{\omega r_{1}}{a}\right) N_{1}^{\prime}\left(\frac{\omega r_{0}}{a}\right) m \frac{1}{r_{0}}\left(\omega^{2}-k_{2}^{2}\right)=0
\end{aligned}
$$

From Eq (26) we can use dimensionless quantities by introducing the following denotations:

$$
\frac{\omega r_{0}}{a}=\bar{\omega}, \quad \bar{k}_{1}=\frac{k_{1} r_{0}}{a}, \quad \bar{k}_{2}=\frac{k_{2} r_{0}}{a}, \quad \bar{k}_{0}=\frac{k_{0} r_{0}}{a}, \frac{\omega r_{1}}{a}=\bar{\omega}_{1}
$$

In essence therefore $\mathrm{Eq}(27)$ takes a simpler form as $\mathrm{Eq}(28)$ expressed as:

$$
\bar{k}=\sqrt{\frac{F_{1}}{F_{2}}}
$$

where $F_{1}$ is expressed as;

$$
\begin{aligned}
& F_{1}=c \bar{\omega}^{4}\left(J_{0}(\bar{\omega}) N_{0}(c \bar{\omega})-J_{0}(c \bar{\omega}) N_{0}(\bar{\omega})\right)+\bar{\omega}^{3}\left(J_{1}(c \bar{\omega}) N_{0}(\bar{\omega})-J_{0}(\bar{\omega}) N_{1}(c \bar{\omega})\right)+ \\
& +c \bar{\omega}^{3}\left(J_{0}(c \bar{\omega}) N_{1}(\bar{\omega})-J_{1}(\bar{\omega}) N_{0}(c \bar{\omega})\right)+\left(J_{1}(\bar{\omega}) N_{1}(c \bar{\omega})-J_{1}(c \bar{\omega}) N_{1}(\bar{\omega})\right)+ \\
& +c \bar{\omega}^{3} m J_{0}(c \bar{\omega}) N_{1}(\bar{\omega})-\bar{\omega}^{2} J_{1}(c \bar{\omega}) N_{1}(\bar{\omega})
\end{aligned}
$$

and $F_{2}$ expressed as; 


$$
\begin{aligned}
& F_{2}=-c \bar{\omega}\left(J_{0}(c \bar{\omega}) N_{0}(\bar{\omega})-J_{1}(c \bar{\omega}) N_{1}(\bar{\omega})\right) m-\left(1+b^{2}\right)\left(\bar{\omega}^{3} c\left(J_{0}(\bar{\omega}) N_{0}(c \bar{\omega})-J_{0}(c \bar{\omega}) N_{0}(\bar{\omega})\right)\right)+ \\
& +\bar{\omega}\left(J_{1}(c \bar{\omega}) N_{0}(\bar{\omega})-J_{0}(\bar{\omega}) N_{1}(c \bar{\omega})\right)+c \bar{\omega}\left(J_{0}(c \bar{\omega}) N_{1}(\bar{\omega})-J_{1}(\bar{\omega}) N_{0}(\bar{\omega})\right)+\left(J_{1}(\bar{\omega}) N_{1}(c \bar{\omega})-J_{1}(c \bar{\omega}) N_{1}(\bar{\omega})\right) \\
& \bar{k}_{1}=b \bar{k}_{2} \quad \bar{k}_{2}=\bar{k} \quad \bar{k}_{1}=b \bar{k} \quad \bar{\omega}_{1}=c \bar{\omega}
\end{aligned}
$$

Eq (28) is a simulation expression connecting the frequency of the system with the free frequency of the any shell without a fluid in it and can be used in finding of the frequencies of eigen oscillations of the system is connected in the whole to the solution of transcendental as expressed in Eq (27). In general approximate methods, simulation show asymptotical behaviuor. However, a numerical solution using the inverse problem allows building the spectrum of graphs using software and this is what simplifies the investigation hence easy to determine frequency when using softwares.

This article was supported by the Department of Mathematics and Physics of Technical University of Mombasa in March 2015.

\section{REFERENCES}

Agalarova T.D. (1997); Interaction of acoustic wave with the oscillator. Collection of scinetific works on Mechanics, 7: 181-184

Chen S.S., Wambsganss M. W., Jendrzejczyk J.A. (1976), Added mass and damping of a vibration rod in confined viscous fluid. Trans. ASME. J.Appl. Mech. 43: 325-329.

Gorshkov A. G., Tarlakovskii D. V. (1990); Unsteady aero hydro elasticity of bodies with spherical form; Phys. And Marh. Met., ISBN-5-20-14006-6.

Huang H., Lu Y.P., Wang Y. (1974); Transient interaction of spherical acoustic waves of a cylindrical elastic shell and its internal multi-degree-of-freedom mechanical systems, J. Acoust. Soc. America, 56: 4-10.

Mamedova G.A, Rustamova M. A., Agasiyev S.P. (2013); Investigation of free oscillations of the spherical shell with fluid by inverse method. Eastern European Journal ofadvancedtechnologies, 66:16-20.

Mosiori, C. (2014). Synthesis Procedures for Silver Nanoparticles. Engineering International, 2(2), 87-90.

Mosiori, C., Maera, J., Njoroge, W., Shikambe, T., Munji, M., \& Magare, R. (2015). Modeling Transfer of Electrons between Energy States of an Electrolyte and CdS thin Films using Gerischer Model. Engineering International, 3(1), 35-44.

Seyfullayev A. I, Rustamova M. A., Agasiev S. R. (2014); Free oscillations of two concentrically located cylindrical shells with a fluid between them. International Journal of Engineering and Innovative Technology , 3: 33-37

Seyfullayev A. I., Mamedova G.A., Rustamova M. A., Yuzbashiyeva A. O. (2012); Analyzes of free oscialltions of thin - walled cylindrical shells containing a compressible liquid. Engen-Phys. Journ., 85:134-149

Seyfullayev A.I., Agayeva N.A. (1998); Solution of the problem on motion of the spherical inclusion with the spring body in the acoustic medium., Phys.-tech-and math. sciences, 18: 133-135.

Sinyavskii V.F, Phedotovskii V.S., Kukhtin A.B. (1980), On vibrations of the cylinder in a viscous liquid. Applied Mechanics, XVI: 62-67.

Online Archive Link: https://abc.us.org/ojs/index.php/ei/issue/archive 